\title{
Dietary Management of Patients with Eosinophilic Esophagitis
}

\section{Alfredo J. Lucendo, MD, FEBGH Ángel Arias, BSC, MSC}

\author{
Corresponding author \\ *Department of Gastroenterology, Hospital General de Tomelloso, Vereda de \\ Socuéllamos, s/n, 13700, Tomelloso, Ciudad Real, Spain \\ Email: alucendo@vodafone.es
}

Published online: 5 February 2014

(C) Springer International Publishing AG 2014

Keywords Eosinophilic esophagitis - Diet - Treatment - Dietary therapy · Food hypersensitivity · Food reintroduction

\section{Opinion statement}

Twenty years after it was first described, eosinophilic esophagitis (EoE) is now recognized as a prevalent disorder, representing the second most common cause of chronic esophagitis (after gastroesophageal reflux) and constituting the main cause of esophageal symptoms in young patients. In most cases the disease is triggered and maintained by exposure to food antigens, thus representing a novel form of food allergy. While topical steroids still constitute the prevailing therapeutic option, there is increased interest in dietary therapy as a treatment alternative capable of inducing and maintaining drug-free remission of the disease. First assessed in a pediatric series, more recent studies have demonstrated that dietary therapy is equally effective for adult sufferers of EoE. Indeed, various types of dietary interventions that either eliminate or reduce food antigens from the diet have been shown to induce remission of esophageal inflammation and its derived symptoms in a high proportion of patients. After remission has been achieved, food reintroduction is an essential part of the dietary management of EoE and should always be considered once esophageal biopsies are normal. Food reintroduction consists of a food allergy challenge that facilitates the identification of specific food triggers for the disease. This allows patients to follow as normal a diet as possible, thus improving the acceptance of and adherence to specific dietary restrictions. The dietary management of EoE is a challenging therapy that should be considered for every patient with the disease. Its major limitations arise from the necessity of repeated endoscopies with biopsies during the food reintroduction protocol, as well as from the absence of noninvasive subrogated markers of disease activity. For these reasons, the use of dietary therapy has been limited to highly motivated patients who are reluctant to utilize drug-based therapies on a chronic basis, as well as to highly motivated health-care providers.0ngoing research on less restrictive dietary options 
should facilitate the spread of dietary interventions, making this type of therapy more available for extended use.

\section{Introduction}

Eosinophilic esophagitis (EoE) represents an emerging gastrointestinal disorder characterized by symptoms of esophageal dysfunction, including dysphagia and food impaction, along with marked esophageal eosinophilia in mucosal biopsies, both of which persist after treatment for acid gastroesophageal reflux [1]. First defined 20 years ago as a distinctive clinico-pathological syndrome [2], EoE currently represents an emerging chronic disease that usually persists from childhood into adulthood $[3 \bullet 4,4]$, with an estimated prevalence of 43-56 cases/100,000 inhabitants in both America and Europe, affecting both children and adults [5-8].

EoE has been related to allergies since its first descriptions. In fact, pediatric and adult patients commonly present a family or personal allergic background, including asthma, rhinitis, conjunctivitis, or eczema with variable frequency [1]. Moreover, peripheral eosinophilia is present in $50 \%$ of patients, with three out of four exhibiting elevated serum IgE levels [9]. Food and aeroallergen sensitization are also common, as determined by means of positive skin prick tests (SPTs) in patients of all ages [10-12]. However, the definitive definition of EoE as a characteristic manifestation of food allergy only came after disease remission was achieved by feeding a series of pediatric patients exclusively with an amino acid-based elemental formula [13]. In this groundbreaking study, the disease recurred in all cases once patients resumed a normal diet, demonstrating unequivocally for the first time that food allergy plays a causal role in chronic esophagitis, while simultaneously establishing a gold standard for comparing subsequent therapies.

However, despite the great number of studies carried out on the disease over the past 20 years, management of EoE remains controversial. Current treatments include the use of swallowed steroids with a topical effect, various dietary modifications, and endoscopic dilation. Even more recently, several monoclonal antibodies have been assayed [14]. With all this, a commonly accepted algorithm for treating patients is still lacking, mostly due to limited information regarding the long-term effects of different therapies in modifying the natural history of the disease and its associated subepithelial fibrosis $[14,15 \bullet, 16 \bullet]$. As a result, there is a wide variability in both the standard of care for EoE patients and the adherence to proposed international guidelines in clinical practice $[6,17 \bullet]$.

An increasing body of knowledge has recently renewed interest in the dietary management of EoE as a drug-free alternative to topical steroids, which still constitute the most widely utilized medical treatment for EoE in patients of all ages. This article aims to review all available dietary approaches for the management of EoE, along with the advantages and limitations of each, in order to provide practical advice for the successful management of EoE through diet.

\section{Treatment}

- The natural history of EoE is starting to become more clearly defined, mainly through longitudinal studies focused on determining the evolution of the untreated disease. We know that, in the absence of treatment, EoE tends to persist over time, with chronic symptoms and inflammation significantly affecting health-related quality of life (QoL) [3•], but usually with no impact on the nutritional state of the patient [18]. Characteristic endoscopic features in children include 
acute inflammation, especially mucosal edema, along with whitish exudates and linear furrows [19]; adult patients additionally present with fibrotic changes, such as rings and strictures [20]. However, no malignant potential has been associated with EoE until now [18]. Moreover, retrospective studies have demonstrated that the duration of untreated disease directly correlates with the prevalence of esophageal strictures in a time-dependent manner [21•]. Likewise, an association has been demonstrated in the natural history of Crohn's disease.

- The classical treatment goals of EoE, namely (1) the resolution of clinical symptoms and (2) achieving and maintaining disease remission, have been expanded to include (3) the prevention of fibrotic complications such as strictures, (4) avoidance of the iatrogenic effects of medication, (5) maintenance of an adequate QoL, and (6) the prevention of nutritional deficiencies due to dietary treatment.

- Pharmacological treatment for EoE patients has mainly comprised the use of several anti-inflammatory drugs used in other allergic disorders. The first reported cases of EoE were effectively managed with systemic steroids, which were later replaced by topical steroids after these were shown to have a similar effectiveness with a lower rate of side effects. First used in pediatric patients [22], topicallyadministered swallowed fluticasone propionate (FP) has been shown to be highly effective in children [23], significantly superior to a placebo [24] and comparable to oral prednisone [25] (1b, grade A). Similar results have been documented in adults [26]. Viscous budesonide has also emerged as an alternative treatment for both children [27] and adults with EoE [28], having been proven safe and effective ( $1 \mathrm{~b}$, grade A). Ciclesonide has also recently been added to the pharmacological armory for treating EoE [29]. However, given the chronic nature of EoE, sustained treatment with steroids or repeated courses must be considered, even after achieving disease remission. Finally, azathioprine/ 6 mercaptopurine was found to be effective in causing the remission of symptoms and eosinophilic infiltration for periods of 3-8 years in adults with steroid-dependent EoE and eosinophilic gastroenteritis with esophageal involvement [30].

- Other anti-allergy drugs such as anti-H1 blockers or the mast cell stabilizer disodium cromoglycate have shown no therapeutic benefit in patients with EoE [31]. Montelukast is likewise not recommended for the management of EoE [1], as it is unable to maintain remission achieved with topical steroids [32] (4, grade C).

- Several biological therapies based on monoclonal antibodies have been assessed in EoE with limited results. Thus, the anti-TNF- $\alpha$ infliximab failed to demonstrate any beneficial effects in adult EoE patients [33], nor did the anti-IgE omalizumab lead to clinical improvement in children [34]. However, when the anti-IL-5 monoclo- 
nal antibodies mepolizumab and reslizumab were analyzed in randomized, controlled trials in both adult [35] and pediatric [36, 37] patients, tissue eosinophils were significantly reduced. Unfortunately, clinical improvement was minimal. (1b, grade A).

- Despite the fact that EoE is defined as the persistence of eosinophilic inflammation after acid suppression, proton pump inhibitors (PPIs) have been used successfully as a sole therapy for EoE patients [38, 39]. The anti-inflammatory properties of PPIs were recently demonstrated in vitro after they were able to reduce the gene expression of eotaxin-3 (a key chemo-attractant involved in the pathophysiology of the disease) in esophageal cell cultures from EoE patients [40]. Until a clearer elucidation of the real significance of PPI-responsive EoE is developed, consensus guidelines currently consider this disease to be a specific manifestation of gastroesophageal reflux disease [1].

- Endoscopic dilation, a mechanical procedure with no effect on the underlying inflammatory process, seems to result in at least a shortterm improvement of symptoms in the majority of patients, according to a recent meta-analysis [41]. It has mostly been used in adult EoE patients, who often present esophageal stenosis with a reduction of esophageal caliber. When performed by an experienced specialist, esophageal dilation is a safe procedure [41]. However, various risk factors for complications have been identified, including a long evolution of dysphagia, high eosinophil density, young patient age, repeated procedures, and luminal narrowing in the upper and middle esophageal thirds [42, 43].

\section{Dietary approaches for EoE}

- Since 1995, after an initial report showed that feeding 10 children exclusively with an amino acid-based elemental formula led to symptom relief and histological normalization of EoE [13], several studies have reproduced these findings in pediatric series [44, 45••, $46 \bullet \bullet$. In fact, results show that more than $90 \%$ of patients rapidly reach peak eosinophil counts $<15 / \mathrm{hpf}$, with symptomatic improvement in $>96 \%$ of cases ( 4 , grade $\mathrm{C}$ ). Moreover, subsequent controlled re-introduction of solid foods results in recurrence of gastrointestinal symptoms specific to individual "trigger" foods.

- The first report regarding the use of elemental diet to treat adult EoE has been published only very recently $[47 \bullet \bullet]$. A series of 29 patients were prospectively recruited and told to avoid any kind of food except an elemental formula for a four-week period. Three patients abandoned the study protocol on the first day and eight more consumed forbidden solid foods during the first 2 weeks of the study period. A pathological infiltration of $>15$ eos/hpf persisted in only one out of the 18 adults who completed the study (4, grade C), giving a per-protocol efficacy of $94.4 \%$, which went down to $58.8 \%$ when analyzed for intention-to treat. 
- A rapid recurrence of eosinophilic inflammation after resuming a normal diet is the norm in both children and adults; therefore, additional dietary strategies should be implemented after elemental diet-induced remission of the disease.

- Although the efficacy of an elemental diet has proven superior to that of any other type of dietary intervention in managing EoE, several drawbacks restrict its use in clinical practice, including its unpleasant taste (which forces up to $80 \%$ of pediatric patients to be fed through a nasogastric tube [31]), its high non-adherence rates [48], the many limitations it places on social activities due to the complete avoidance of any kind of table food, and its high cost. This latter limitation leads to the additional disadvantage that some insurance plans do not cover this type of therapy.

- The only realistic utility of elemental diet in clinical practice is to feed infants and toddlers, among whom the restriction of having no additional food may be better tolerated, and only during the length of time required for food reintroduction with the goal of identifying specific dietary triggers.

\section{Elimination diets directed by allergy testing}

- The growing body of evidence showing that exposure to food allergens leads to EoE has prompted researchers to attempt to identify specific food triggers for the disease, sometimes using clinical histories (which is complicated as patients do not usually associate the consumption of certain foods with the appearance of symptoms), but most often using skin prick tests (SPTs) and/or atopy patch tests (APTs).

- In 2002, Spergel et al. used SPTs and APTs on pediatric EoE patients for the first time to determine a suitable elimination diet [49]. The avoidance of foods that gave positive skin test results led to histological and clinical resolution in $49 \%$ of patients [50•]; with this strategy, an average of five foods were excluded from each child's diet ( 4 , grade $\mathrm{C}$ ). The authors adverted on the variable sensitivity and specificity of these allergy tests, with $<10 \%$ of positive results in both tests, along with their low sensitivity to cow's milk, which, as noted below, is the most common food trigger for EoE. The same authors have recently evaluated a new strategy consisting of the elimination from the diet of foods identified through SPT/APT, in addition to an empirical elimination diet. Combining the two strategies led to increases in histological remission rates of up to $77 \%$ [50•].

- Unfortunately, the aforementioned results regarding the histological remission of EoE have not been reproduced by other research groups. Allergy tests failed to identify food triggers in most patients in several pediatric $[51 \bullet, 52,53]$ and adult series $[47 \bullet \bullet, 54 \bullet \bullet, 55,56 \bullet, 57 \bullet \bullet]$. Moreover, food-specific IgE serum measurements and SPTs were 
neither sensitive nor specific methods for predicting EoE triggers in adult patients $[57 \bullet \bullet, 58 \bullet \bullet]$.

- A growing body of evidence points to the involvement of a cellmediated delayed reaction against foods rather than an IgE-mediated reaction in the pathophysiology of EoE. As a result, EoE usually coexists together with other IgE-mediated atopic manifestations in a single patient, each of which responds to different underlying mechanisms, thus limiting the clinical utility of IgE testing for directing dietary interventions in patients with EoE. Indeed, because positive results from SPTs, serum IgE, and ATPs cannot be used alone to identify food triggers in EoE, food restrictions should not be recommended exclusively based on a positive result. Currently, food triggers can only be identified by documenting disease remission after specific food antigen avoidance, followed by EoE recrudescence upon reintroduction of the food in question [1]. This is the strategy used in the empirical elimination diets and food reintroduction protocols discussed below.

\section{Empirical elimination diets in EoE}

- In an attempt to overcome the disadvantages of elemental and allergytesting directed diets in children with EoE, Kagalwalla et al. explored the alternative of eliminating the six foods containing those intact food proteins most commonly associated with food allergy in children [45••]. The original 6-food elimination diet (SFED), which specifically excluded milk protein, soy, egg, wheat, peanut/tree nuts, and seafood from the subjects' diets, led to significant improvement of both esophageal inflammation $(<10 \mathrm{eos} / \mathrm{hpf})$ and symptoms in $74 \%$ of the 35 children treated during a 6week period.

- Comparable response rates to those reported in children [46••] have also been documented for SFED in two prospective American and

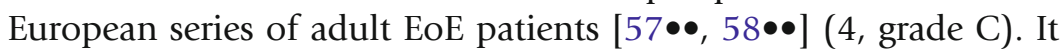
should be noted that the original list of foods restricted by Kagalwalla was broadened to include foods that gave a positive SPT result $[46 \bullet \bullet, 59 \bullet \bullet]$, taking into account geographical food sensitization patterns $[58 \bullet \bullet]$.

- Most importantly, using sequential single food reintroduction, researchers were able to clearly identify individual food triggers in chil-

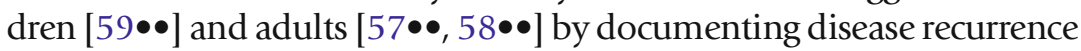
through repeated endoscopies with biopsies. Cow's milk, wheat, and eggs (in that order) have been identified as the major food triggers in all available studies to date. The frequency and involvement of the remaining triggers varies from one region to another, giving rise to the question of whether empirical exclusion diets should be tailored to each specific region and based on the staple diets and food-sensitization 


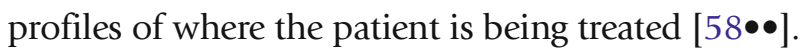

- The sustained efficacy of food trigger avoidance has only been assessed in two studies $[58 \bullet \bullet, 60]$. For a period of up to 3 years, every patient who followed the diet remained asymptomatic, with no pathological eosinophilic inflammation seen in annual endoscopic examinations. Moreover, no drug treatment was necessary for pa-

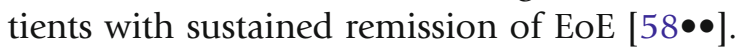

- Finally, allergy tests based on demonstrating an IgE-driven hypersensitivity showed limited usefulness in identifying EoE triggers in SFED-based studies, exhibiting extremely low concordance with the

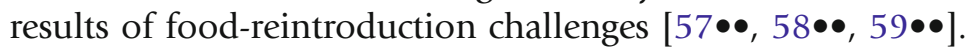

\section{Empirical elimination of cow's milk}

- Cow's milk protein has been demonstrated to be the food antigen

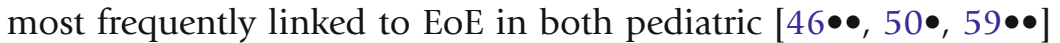
and adult patients, $[57 \bullet \bullet, 58 \bullet \bullet]$ identified as an EoE trigger in approximately three out of four subjects.

- There are an increasing number of reports of patients developing EoE after milk [61] and egg [62] oral immunotherapy. Data on long-term food tolerance induction are still scarce [63]; the possibility of a change in the pattern of the immune response from Th2 to Th1-type should be taken into account.

- As a result, a recent retrospective study [64•] focusing solely on cow's milk showed significant histological remission of EoE ( $<15$ eos/hpf) in $65 \%$ of children and symptom improvement in all patients after the elimination of milk from the diet. The unexpectedly high efficacy of this strategy may have been influenced in this study by the inclusion of patients with a particular allergic background, i.e., a previous IgE-mediated cow's milk allergy undergoing desensitization.

- In conclusion, EoE patients with a previous history of Th2-mediated food allergy and who are undergoing oral desensitization should be considered for specific single-food removal, followed by endoscopic and bioptic monitoring for EoE remission. The advantages and drawbacks of re-exposing the patient to allergic reaction, including anaphylaxis in the case of accidental consumption, should be carefully weighed.

\section{Food reintroduction and identification of food triggers}

- Food reintroduction is essential in the dietary management of EoE and should always be considered after patients on elemental or elimination diets present normal esophageal biopsies. Food reintroduction has the double aim of selectively identifying foods that trigger EoE, as well as improving patient acceptance of and adherence to a less restrictive diet. 
- It is worth noting that one or more independent foods can be responsible for EoE; thus, while three out of four Northwestern American (pediatric and adult) patients exhibited a single food trigger $[57 \bullet \bullet, 59 \bullet \bullet]$, this was documented in only one out of three adult Spanish patients $[58 \bullet \bullet$. Although very limited information is available on this topic, following a more varied diet (the Mediterranean diet as compared to the Northwestern American diet or an adult's diet compared to a child's), may increase the probability of developing a food allergy manifested as EoE.

- There are two possible strategies for planning the reintroduction sequence. The first is to begin with foods unlikely to cause EoE (e.g., vegetables and fruits, chicken and beef), in order to normalize the patient's diet as soon as possible [65]. The alternative strategy, assessed in SFED-based trials, consists of first reintroducing wheat and milk, because although they are the most common EoE triggers, if the result is negative, their impact in returning patients to a normal diet is higher $[58 \bullet \bullet]$.

- After demonstrating EoE triggered by cow's milk, the immediate reintroduction of soy may provide patents with an effective milk-substitute. Due to the common cross-reactivity with cow's milk proteins, sheep's and goat's milk-derived products (mainly cheeses) should also be excluded.

\section{How can we succeed in the dietary management of EoE patients?}

- The lack of multicenter, randomized, comparative studies on the effectiveness, advantages and drawbacks of empirical elimination diets vs. skin allergy testing prevent us from undoubtedly recommending one dietary option over another. However, available evidences show that SFED seems to be the best current dietary approach for treating EoE patients, having provided the greater and more consistent results in different studies, and it should be considered for both children and motivated adults. Until better and more accurate food allergy testing capable of identifying specific food triggers is available or until genetic profiling can accurately predict individual responses to diet, the unreliability of skin allergy testing limits the use of this method to experienced centers where it has been proven to be efficient.

- Food triggers of EoE have repeatedly been demonstrated to be foods commonly consumed in the standard Western diet, including milk, wheat, and eggs. This begs the question of whether these trigger foods are the same for other geographical regions, for example, Asia, where rice and soy are common in the staple diet. Consequently, when planning a dietary intervention to manage EoE, it should be taken into account that allergy sensitization patterns depend on antigen exposure, which varies from one place to another [66-68]. 
- As a result, it seems reasonable to design food interventions based on skin allergy testing or empirical elimination according to the staple diet, adapting each strategy to regional consumption habits and allergy sensitization patterns.

- To date, there is no definitive data on the specific predictors of clinical response to dietary intervention in adult EoE; indeed, neither symptoms nor endoscopic/histopathological background have dif-

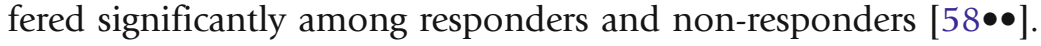
Moreover, extended experience in managing adult EoE with an SFED was associated with increased response rates $(p<0.05)[57 \bullet \bullet]$. In the case of exclusive elimination of cow's milk, younger patients and those with a lower peak eosinophil count exhibited a significantly increased remission rate $[64 \bullet]$.

- The risk of nutritional deficiencies derived from extensive food restrictions must be adverted, especially in pediatric EoE patients. The collaboration of a dietician is strongly recommended for patient and parent dietary counseling, as a specialist can provide useful instructions and guidelines to ensure correct dietary management of the disease. The possibly harmful effects of food restrictions on adult patients seem to be minimal. In any case, extended food restrictions like those imposed in empirical SFEDs should only be used to induce disease remission over the course of several weeks. After that, food reintroduction constitutes an essential part of the dietary management of EoE patients, and should be always considered after normal esophageal biopsies have been achieved.

- Long-term difficulties in adhering to an elimination diet mostly depend on the type and number of food triggers involved in EoE. Key factors for improving adherence include finding appropriate substitutes for the eliminated foods and simplifying the food elimination study protocols. Cow's milk-based, extensively hydrolyzed formulas have been demonstrated to be well-tolerated by most adult patients with milk-triggered EoE [69•]. Ongoing research on 4 -food elimination diets may simplify study protocols and reduce the number of endoscopies needed.

- Since repeated endoscopies and biopsies are essential for identifying EoE food triggers, sedation should be guaranteed for these patients, especially since it increases their willingness to undergo repeated procedures. In order to reduce the number of endoscopic exams needed, some authors have performed them after the reintroduction of two foods, but this strategy may lead to potential confusion, since symptoms do not universally reappear quickly in every patient.

\section{Unsolved aspects \& suggestions for further research}

- It is interesting to note that the various dietary interventions available for EoE patients show relatively similar results with regard to 
efficacy, ranging from 96 to $70 \%[13,32,44,45 \bullet \bullet, 46 \bullet \bullet, 50 \bullet, 57 \bullet \bullet$, $58 \bullet \bullet$. We can thus safely assert that in most of patients with EoE, the disease is triggered and maintained exclusively by food, with a very small remainder attributable to airborne allergens.

- Differences in the efficacy of exclusive feeding with an elemental diet compared with empirical elimination diets can be explained by allergy to those foods that are not restricted from the diet, mostly fruits and vegetables. In fact, most adult EoE patients exhibit sensitization to cross-reactive panallergen components, including profilins [70] and lipid transfer proteins (LPT), which are mostly found in food of vegetal origin.

- Because EoE is a chronic malady, foods identified as triggers for the disease should be avoided indefinitely [1]; after that, and even when a sustained drug-free response is achievable for most patients with EoE, it is tempting to speculate on the possibility of inducing food tolerance by progressive EoE trigger reintroduction. Unfortunately, the only available study on this topic provided

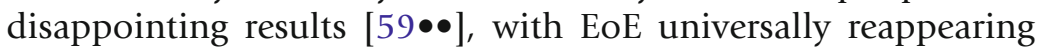
after food reintroduction in every child who had been in remission for a period of up to 4 years.

- The search for noninvasive markers of active esophageal inflammation must go on in order to facilitate the use of dietary therapies for EoE. Unfortunately, subrogate biochemical markers, including serum levels of eosinophil-derived granular proteins, have shown little usefulness in monitoring disease activity [71]. However, a novel, minimally invasive string test was recently found to accurately reflect mucosal eosinophilic inflammation by measuring eosinophil-derived proteins in luminal secretions [72]. Further research is needed to demonstrate the usefulness of this test in clinical practice.

- Finally, in order to better define novel and targeted dietary-based therapies, the many unresolved aspects concerning the immunological and physiopathological mechanisms of EoE must be elucidated.

\section{Compliance with Ethics Guidelines}

\section{Conflict of Interest}

Alfredo J Lucendo and Ángel Arias declare that they have no conflicts of interest.

Human and Animal Rights and Informed Consent

This article does not contain any studies with human or animal subjects performed by any of the authors. 


\section{References and Recommended Reading}

Papers of particular interest, published recently, have been highlighted as:

- Of importance

$\bullet \quad$ Of major importance

1. Liacouras CA, Furuta GT, Hirano I, et al. Eosinophilic esophagitis: updated consensus recommendations for children and adults. J Allergy Clin Immunol. 2011;128:3-20.

2. Attwood SE, Smyrk TC, Demeester TR, et al. Esophageal eosinophilia with dysphagia. A distinct clinicopathologic syndrome. Dig Dis Sci. 1993;38:109-16.

3. DeBrosse CW, Franciosi JP, King EC, et al. Long-term outcomes in pediatric-onset esophageal eosinophilia. J Allergy Clin Immunol. 2011;128:132-8.

After retrospectively identifying EoE patients by reviewing pediatric esophageal biopsy specimens from up to 1999, they were contacted 15 years later and answered questionnaire-based study; quality of life was significantly decreased among patients compared with control subjects, while EoE symptoms persisted along the time.

4. Lucendo AJ, Sánchez-Cazalilla M. Adult versus pediatric eosinophilic esophagitis: important differences and similarities for the clinician to understand. Expert Rev Clin Immunol. 2012;8:733-45.

5. Noel RJ, Putnam PE, Rothenberg ME. Eosinophilic esophagitis. N Engl J Med. 2004;351:940-1.

6. Dellon ES, Jensen ET, Martin CF, et al. The prevalence of eosinophilic esophagitis in the United States. Clin Gastroenterol Hepatol. 2013. doi:10.1016/ j.cgh.2013.09.008.

7. Hruz P, Straumann A, Bussmann C, et al. Escalating incidence of eosinophilic esophagitis: a 20 -year prospective, population-based study in Olten County, Switzerland. J Allergy Clin Immunol. 2011;128:1349-50.

8. Arias A, Lucendo AJ. Prevalence of eosinophilic oesophagitis in adult patients in a central region of Spain. Eur J Gastroenterol Hepatol. 2013;25:208-12.

9. Dominguez Jimenez JL, Cerezo RA, Marin Moreno MA, et al. Could be possible to predict eosinophil accumulation in esophageal mucosa in eosinophilic esophagitis without perform endoscopic examination? Rev Esp Enferm Dig. 2011;103:385-6.

10. DeBrosse CW, Collins MH, Buckmeier Butz BK, et al. Identification, epidemiology, and chronicity of pediatric esophageal eosinophilia, 1982-1999. J Allergy Clin Immunol. 2010;126:112-9.

11. Roy-Ghanta S, Larosa DF, Katzka DA. Atopic characteristics of adult patients with eosinophilic esophagitis. Clin Gastroenterol Hepatol. 2008;6:531-5.

12. Castro JA, Gomez TE, Garcia RR, et al. Demographic, clinical and allergological characteristics of Eosinophilic Esophagitis in a Spanish central region. Allergol Immunopathol (Madr ), doi: 10.1016/ j.aller.2013.04.004.

13. Kelly KJ, Lazenby AJ, Rowe PC, et al. Eosinophilic esophagitis attributed to gastroesophageal reflux: improvement with an amino acid-based formula. Gastroenterology. 1995;109:1503-12.

14. Gonzalez-Castillo S, Arias A, Lucendo AJ. Treatment of eosinophilic esophagitis: how should we manage the disease? J Clin Gastroenterol. 2010;44:663-71.

15. Lieberman JA, Morotti RA, Konstantinou GN, et al. Dietary therapy can reverse esophageal subepithelial fibrosis in patients with eosinophilic esophagitis: a historical cohort. Allergy. 2012;67:1299-307.

A retrospective analysis of a biopsy database showed that dietary restriction alone, similar to topical steroids, can reverse subepithelial fibrosis in children with EoE.

16. Lucendo AJ, Arias A, De Rezende LC, et al. Subepithelial collagen deposition, profibrogenic cytokine gene expression, and changes after prolonged fluticasone propionate treatment in adult eosinophilic esophagitis: a prospective study. J Allergy Clin Immunol. 2011;128:1037-46.

The first assessment of fibrous remodeling in adult patients with EoE demonstrated a dense subepithelial collagen deposition; prolonged treatment with fluticasone propionate did not significantly reduced this deposit, in spite of reducing gene expression of pro-fibrogenic cytokines.

17. Lucendo AJ, Arias A, Molina-Infante J, et al. Diagnostic and therapeutic management of eosinophilic oesophagitis in children and adults: Results from a Spanish registry of clinical practice. Dig Liver Dis. 2013;45:562-8.

A nationwide prospective survey-based registry of pediatric and adult patients with EoE documented a wide variability in clinical practice regarding diagnostic and therapeutic management of patients. The physician experience and the availability of hospital facilities were the major explicative factors.

18. Straumann A, Spichtin HP, Grize L, et al. Natural history of primary eosinophilic esophagitis: a followup of 30 adult patients for up to 11.5 years. Gastroenterology. 2003;125:1660-9. 
19. Fox VL. Eosinophilic esophagitis: endoscopic findings. Gastrointest Endosc Clin N Am. 2008;18:4557.

20. Straumann A, Aceves SS, Blanchard C, et al. Pediatric and adult eosinophilic esophagitis: similarities and differences. Allergy. 2012;67:477-90.

21. Schoepfer AM, Safroneeva, Bussmann C, et al. Delay in Diagnosis of Eosinophilic Esophagitis Increases Risk for Stricture Formation, in a TimeDependent Manner. Gastroenterology.

2013;145:1230-6.

A retrospective analysis of a database demonstrated that the duration of an untreated disease directly correlated with the prevalence of esophageal fibrotic strictures in adult EoE patients.

22. Faubion Jr WA, Perrault J, Burgart LJ, et al. Treatment of eosinophilic esophagitis with inhaled corticosteroids. J Pediatr Gastroenterol Nutr. 1998;27:90-3.

23. Teitelbaum JE, Fox VL, Twarog FJ, et al. Eosinophilic esophagitis in children: immunopathological analysis and response to fluticasone propionate. Gastroenterology. 2002;122:1216-25.

24. Konikoff MR, Noel RJ, Blanchard C, et al. A randomized, double-blind, placebo-controlled trial of fluticasone propionate for pediatric eosinophilic esophagitis. Gastroenterology. 2006;131:1381-91.

25. Schaefer ET, Fitzgerald JF, Molleston JP, et al. Comparison of oral prednisone and topical fluticasone in the treatment of eosinophilic esophagitis: a randomized trial in children. Clin Gastroenterol Hepatol. 2008;6:165-73.

26. Lucendo AJ, Navarro $M$, Comas C, et al. Immunophenotypic characterization and quantification of the epithelial inflammatory infiltrate in eosinophilic esophagitis through stereology: an analysis of the cellular mechanisms of the disease and the immunologic capacity of the esophagus. Am J Surg Pathol. 2007;31:598-606.

27. Aceves SS, Bastian JF, Newbury RO, et al. Oral viscous budesonide: a potential new therapy for eosinophilic esophagitis in children. Am J Gastroenterol. 2007;102:2271-9.

28. Straumann A, Conus S, Degen L, et al. Budesonide is effective in adolescent and adult patients with active eosinophilic esophagitis. Gastroenterology. 2010;139:1526-37.

29. Schroeder S, Fleischer DM, Masterson JC, et al. Successful treatment of eosinophilic esophagitis with ciclesonide. J Allergy Clin Immunol. 2012;129:1419-21.

30. Netzer P, Gschossmann JM, Straumann A, et al. Corticosteroid-dependent eosinophilic oesophagitis: azathioprine and 6-mercaptopurine can induce and maintain long-term remission. Eur J Gastroenterol Hepatol. 2007;19:865-9.

31. Liacouras CA, Spergel JM, Ruchelli E, et al. Eosinophilic esophagitis: a 10-year experience in 381 children. Clin Gastroenterol Hepatol. 2005;3:1198-206.

32. Lucendo AJ, De Rezende LC, Jimenez-Contreras $S$, et al. Montelukast was inefficient in maintaining steroid-induced remission in adult eosinophilic esophagitis. Dig Dis Sci. 2011;56:3551-8.

33. Straumann A, Bussmann C, Conus S, et al. Anti-TNFalpha (infliximab) therapy for severe adult eosinophilic esophagitis. J Allergy Clin Immunol. 2008; 122:425-7.

34. Rocha $\mathrm{R}$, Vitor $\mathrm{AB}$, Trindade $\mathrm{E}$, et al. Omalizumab in the treatment of eosinophilic esophagitis and food allergy. Eur J Pediatr. 2011;170:1471-4.

35. Straumann A, Conus S, Grzonka P, et al. Anti-interleukin-5 antibody treatment (mepolizumab) in active eosinophilic oesophagitis: a randomised, placebo-controlled, double-blind trial. Gut. 2010;59:21-30.

36. Assa'ad AH, Gupta SK, Collins MH, et al. An antibody against IL-5 reduces numbers of esophageal intraepithelial eosinophils in children with eosinophilic esophagitis. Gastroenterology. 2011;141:1593-604.

37. Spergel JM, Rothenberg ME, Collins $\mathrm{MH}$, et al. Reslizumab in children and adolescents with eosinophilic esophagitis: results of a double-blind, randomized, placebo-controlled trial. J Allergy Clin Immunol. 2012;129:456-63.

38. Peterson KA, Thomas KL, Hilden K, et al. Comparison of esomeprazole to aerosolized, swallowed fluticasone for eosinophilic esophagitis. Dig Dis Sci. 2010;55:1313-9.

39. Molina-Infante J, Ferrando-Lamana L, Ripoll C, et al. Esophageal eosinophilic infiltration responds to proton pump inhibition in most adults. Clin Gastroenterol Hepatol. 2011;9:110-7.

40. Cheng E, Zhang X, Huo X, et al. Omeprazole blocks eotaxin-3 expression by oesophageal squamous cells from patients with eosinophilic oesophagitis and GORD. Gut. 2013;62:824-32.

41. Moawad FJ, Cheatham JG, Dezee KJ. Meta-analysis: the safety and efficacy of dilation in eosinophilic oesophagitis. Aliment Pharmacol Ther. 2013;38:713-20.

42. Cohen MS, Kaufman AB, Palazzo JP, et al. An audit of endoscopic complications in adult eosinophilic esophagitis. Clin Gastroenterol Hepatol. 2007;5:1149-53.

43. Dellon ES, Gibbs WB, Rubinas TC, et al. Esophageal dilation in eosinophilic esophagitis: safety and predictors of clinical response and complications. Gastrointest Endosc. 2010;71:706-12. 
44. Markowitz JE, Spergel JM, Ruchelli E, et al. Elemental diet is an effective treatment for eosinophilic esophagitis in children and adolescents. Am J

Gastroenterol. 2003;98:777-82.

45.• Kagalwalla AF, Sentongo TA, Ritz S, et al. Effect of sixfood elimination diet on clinical and histologic outcomes in eosinophilic esophagitis. Clin Gastroenterol Hepatol. 2006;4:1097-102.

The first demonstration that the empiric exclusion of the most common food allergens led to remission of EoE in pediatric patients.

46.• Henderson CJ, Abonia JP, King EC, et al. Comparative dietary therapy effectiveness in remission of pediatric eosinophilic esophagitis. J Allergy Clin Immunol. 2012;129:1570-8.

The efficacy of the three major dietary-based therapies was compared in this retrospective pediatric cohort analysis; elemental diet was superior in inducing histologic remission compared with six-food elimination and skin tests-directed diets, these two last alternatives being equally effective.

47.•• Peterson KA, Byrne KR, Vinson LA, et al. Elemental diet induces histologic response in adult eosinophilic esophagitis. Am J Gastroenterol. 2013;108:759-66.

This is the only study that assesses the efficacy of exclusive feeding with an elemental diet in adult patients. Disease remitted in $95 \%$ of the patients who completed the protocol, but the adherence to the diet constituted a relevant problem.

48. Hommel KA, Franciosi JP, Gray WN, et al. Behavioral functioning and treatment adherence in pediatric eosinophilic gastrointestinal disorders. Pediatr Allergy Immunol. 2012;23:494-9.

49. Spergel JM, Beausoleil JL, Mascarenhas M, et al. The use of skin prick tests and patch tests to identify causative foods in eosinophilic esophagitis. J Allergy Clin Immunol. 2002;109:363-8.

50. Spergel JM, Brown-Whitehorn TF, Cianferoni A, et al. Identification of causative foods in children with eosinophilic esophagitis treated with an elimination diet. J Allergy Clin Immunol. 2012;130:461-7.

A large retrospective analysis of 941 children with EoE attended in a single center during an 11-year period. Both empirical elimination diets and skin allergy-based food exclusion resulted equally effective if cow's milk was also removed in addition foods with a positive SPT/APT result.

51. Paquet B, Begin P, Paradis L, et al. Variable yield of allergy patch testing in children with eosinophilic esophagitis. J Allergy Clin Immunol. 2013;131:613. The authors reported their disappointing experience with APT, which were not sensible methods in pediatric EoE, questioning the utility of this test in studying EoE food triggers, which reinforce the superiority of empiric six-food elimination diets.

52. Rizo Pascual JM, De La Hoz CB, Redondo VC, et al. Allergy assessment in children with eosinophilic esophagitis. J Investig Allergol Clin Immunol. 2011;21:59-65.
53. Assa'ad AH, Putnam PE, Collins MH, et al. Pediatric patients with eosinophilic esophagitis: an 8-year follow-up. J Allergy Clin Immunol. 2007;119:731-8.

54.• Molina-Infante J, Martin-Noguerol E, Varado-Arenas $\mathrm{M}$, et al. Selective elimination diet based on skin testing has suboptimal efficacy for adult eosinophilic esophagitis. J Allergy Clin Immunol. 2012;130:1200-2.

The best documented study on the utility of skin allergy testdirected food removal in adult EoE patients; the exclusion of foods with SPT, prick-prick tests, and APT results was only shown effective in $18 \%$ of patients, but after usually excluding many different foods.

55. Simon D, Straumann A, Wenk A, et al. Eosinophilic esophagitis in adults-no clinical relevance of wheat and rye sensitizations. Allergy. 2006;61:1480-3.

56. Gonzalez-Cervera J, Angueira T, RodriguezDominguez B, et al. Successful food elimination therapy in adult eosinophilic esophagitis: not all patients are the same. J Clin Gastroenterol. 2012;46:855-8.

This case series report detailed analysis of three EoE patients who successfully resolved their disease after following different dietary strategies, suggesting that individual differences may justify distinct management strategies.

57.• Gonsalves N, Yang GY, Doerfler B, et al. Elimination diet effectively treats eosinophilic esophagitis in adults; food reintroduction identifies causative factors. Gastroenterology. 2012;142:1451-9.

The first demonstration of the effectiveness of a six-food elimination diet in American adult patients with EoE; an equivalent response rate and similar food triggers to those described in children were reported

58.• Lucendo AJ, Arias A, Gonzalez-Cervera J, et al. Empiric 6-food elimination diet induced and maintained prolonged remission in patients with adult eosinophilic esophagitis: a prospective study on the food cause of the disease. J Allergy Clin Immunol. 2013;131:797-804.

The first six-food elimination diet-based study carried out in a large series of European patients documented equivalent effectiveness to that reported from American research, but different distribution of frequencies of food triggers. The strategy of adapting food restrictions to the regional staple diet and sensitization patterns was suggested for authors. Patients who continuously avoided food triggers maintained prolonged disease remission for up to 3 years.

$59 . \bullet$ Kagalwalla AF, Shah A, Li BU, et al. Identification of specific foods responsible for inflammation in children with eosinophilic esophagitis successfully treated with empiric elimination diet. J Pediatr Gastroenterol Nutr. 2011;53:145-9.

The first demonstration that sequential food reintroduction monitored with repeated endoscopies and biopsies identifies specific food triggers for EoE came from this retrospective research on pediatric population. 
60. Gonsalves N, Doerfler B, Hirano I. Long term maintenance therapy with dietary restriction in adults with eosinophilic esophagitis [abstract]. Gastroenterology. 2011;140(5 Suppl 1):S-180-1.

61. Sanchez-Garcia S, Del Rodriguez RP, Escudero C, et al. Possible eosinophilic esophagitis induced by milk oral immunotherapy. J Allergy Clin Immunol.

2012;129:1155-7.

62. Ridolo E, De Angelis GL, Dall'aglio P. Eosinophilic esophagitis after specific oral tolerance induction for egg protein. Ann Allergy Asthma Immunol. 2011;106:73-4.

63. Savilahti EM, Savilahti E. Development of natural tolerance and induced desensitization in cow's milk. Pediatr Allergy Immunol. 2013;24:114-21.

64. Kagalwalla AF, Amsden K, Shah A, et al. Cow's Milk Elimination: A Novel Dietary Approach to Treat Eosinophilic Esophagitis. J Pediatr Gastroenterol Nutr. 2012;55:711-6.

A retrospective analysis of pediatric EoE patients documented that the exclusion of the single food related to causing EoE resulted in a highly efficient treatment strategy. 65. Spergel JM, Shuker M. Nutritional management of eosinophilic esophagitis. Gastrointest Endosc Clin N Am. 2008;18:179-94.

66. Rona RJ, Keil T, Summers C, et al. The prevalence of food allergy: a meta-analysis. J Allergy Clin Immunol. 2007;120:638-46.
67. Roodenburg AJ, Schlatmann A, Dötsch-Klerk M, et al. Potential effects of nutrient profiles on nutrient intakes in the Netherlands, Greece, Spain, USA, Israel, China and South-Africa. PLoS One. 2011;23:e14721.

68. Vereda A, van Hage M, Ahlstedt S, Ibañez MD, Cuesta-Herranz J, van Odijk J, et al. Peanut allergy: clinical and immunologic differences among patients from 3 different geographic regions. J Allergy Clin Immunol. 2011;127:603-7.

69.• Lucendo AJ, Arias A, González-Cervera J, et al. Tolerance of a cow's milk-based hydrolyzed formula in patients with eosinophilic esophagitis triggered by milk. Allergy. 2013;68:1065-72.

Most of adult patients with EoE triggered by milk tolerated a cow's milk-based hydrolyzed formula, providing them with a safe alternative to substitute cow's milk in the diet.

70. Simon D, Straumann A, Dahinden C, et al. Frequent sensitization to Candida albicans and profilins in adult eosinophilic esophagitis. Allergy. 2013;68:945-8.

71. Rodríguez-Sánchez J, Gómez Torrijos E, de la Santa Belda E, et al. Serological markers of activity in eosinophilic esophagitis. Is this possible? Rev Esp Enferm Dig. 2013;105:462-8.

72. Furuta GT, Kagalwalla AF, Lee JJ, et al. The oesophageal string test: a novel, minimally invasive method measures mucosal inflammation in eosinophilic oesophagitis. Gut. 2013;62:1395-405. 